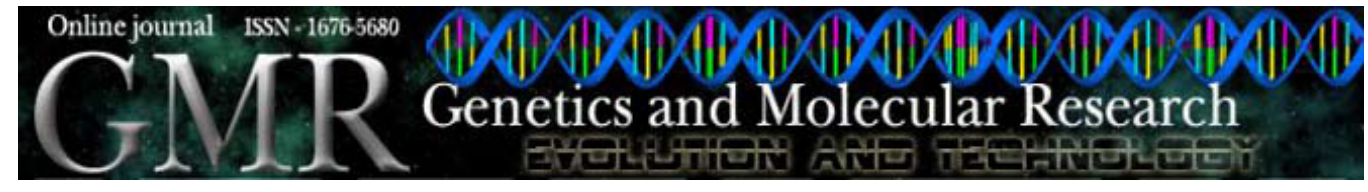

\title{
Decoherence in yeast cell populations and its implications for genome-wide expression noise
}

\author{
M.R.S. Briones and F. Bosco \\ Departamento de Microbiologia, Imunologia e Parasitologia, \\ Universidade Federal de São Paulo, São Paulo, SP, Brasil \\ Corresponding author: F. Bosco \\ E-mail: fbosco@unifesp.br
}

Genet. Mol. Res. 8 (1): 47-51 (2009)

Received September 19, 2008

Accepted November 11, 2008

Published January 20, 2009

\begin{abstract}
Gene expression "noise" is commonly defined as the stochastic variation of gene expression levels in different cells of the same population under identical growth conditions. Here, we tested whether this "noise" is amplified with time, as a consequence of decoherence in global gene expression profiles (genome-wide microarrays) of synchronized cells. The stochastic component of transcription causes fluctuations that tend to be amplified as time progresses, leading to a decay of correlations of expression profiles, in perfect analogy with elementary relaxation processes. Measuring decoherence, defined here as a decay in the auto-correlation function of yeast genome-wide expression profiles, we found a slowdown in the decay of correlations, opposite to what would be expected if, as in mixing systems, correlations decay exponentially as the equilibrium state is reached. Our results indicate that the populational variation in gene expression (noise) is a consequence of temporal decoherence, in which the slow decay of correlations is a signature of strong interdependence of the transcription dynamics of different genes.
\end{abstract}

Key words: Decoherence; Gene expression noise; Transcription; Cell biology; Biophysics; Mathematical biology 


\section{INTRODUCTION}

The variation of gene expression levels in different cells of the same population, under identical growth conditions, has a stochastic component referred to as "noise in gene expression" (Raser and O'Shea, 2005; Paulsson, 2005). The origin of gene expression fluctuations (of populational nature) has been studied using different approaches ranging from phenomenological equations to stochastic equations (Paulsson, 2005; Nacher and Akutsu, 2006). Some authors propose that the fluctuations of gene expression have distinguishable intrinsic and extrinsic sources, where the first is related to the actual synthesis processes (transcription and translation) and the second relates to other sources of fluctuations that could interfere constructively or destructively with the expression process. All these approaches have the same goal of identifying the simplest set of statements to develop a reasonable theory about the cell-to-cell expression fluctuation phenomenon.

Here, we tested whether gene expression noise is amplified with time and therefore could be understood as a consequence of decoherence in global gene expression profiles. Because the chemical process of transcription has a stochastic component, the corresponding fluctuations tend to be amplified as time progresses, leading to a decay of correlations of the global gene expression profile, in perfect analogy with elementary relaxation processes. A crucial question concerns the characterization of the specific stochastic process that models the phenomenon of decay in that case. In standard relaxation, an initial non-equilibrium state decorrelates with time as the system approaches its statistical equilibrium state. If correlations decay exponentially (as in mixing systems) the equilibrium state is reached fast enough so that it is observed in a reasonable time scale. If the system has any internal source of additional information that represents internal organization of the system states, then a slowdown in the decay of correlations is expected. This is exactly what was observed here in the case of global transcriptional gene expression profiles of synchronized cells as described below, where decoherence was measured as the decay of the auto-correlation function of the yeast whole-genome expression profiles (microarrays). The phenomenon of decoherence occurs when the interaction between a system and its surrounding environment is thermodynamically irreversible. One way to describe it is via the decay of time correlation functions.

\section{MATERIAL AND METHODS}

We defined our system as the set of all mRNA molecules present in the cell at a given moment and everything else, external to the system. This system is open to mass and energy exchange and is therefore far from thermodynamic equilibrium. Decoherence was measured using temporal profiles of mRNA expression levels on microarray data of synchronized yeast cell cultures (Saccharomyces cerevisiae) to minimize the delay effects in the cell cycle between individual cells, a possible source of cell-to-cell variation in expression levels (http://cellcycle-www.stanford.edu/) (Cho et al., 1998). The measurements consist of 17 sequential whole-genome microarrays (6220 open reading frames) with time points 7 min apart over 1.8 cell cycles. Cell culture synchronization conveniently guarantees that the temporal development of the global expression profile effectively reveals the existence of undamped fluctuations. 


\section{RESULTS AND DISCUSSION}

The cell cycle-regulated genes (Spellman et al., 1998) were analyzed separately from the whole genome to show the behavior of the correlation function across the cell cycle phases and to show that even for this set of tightly controlled genes, the correlation function of global expression profile also decays. To obtain the correlation functions, we constructed the mean distribution of expression levels for each instantaneous array calculated over several fitting curves, obtaining the approximate density functions $\rho_{t_{i}}(E), i=0,1, \ldots, 16$, corresponding to 17 microarray time point measurements per gene (Cho et al., 1998). The value $i=0$ (relative to the array measurement) is the reference state measured at $t_{0}=0$, such that the correlation function is obtained numerically by

$$
C\left(t_{j}\right)=\int \rho_{t_{j}}(E) \rho_{t_{0}}(E) d E / \int \rho_{t_{0}}(E) \rho_{t_{0}}(E) d E
$$

where the variable $E$ is the instantaneous expression level of genes in the whole-genome microarrays.

The typical variation of the values of $C(t)$ is of the order $10^{-2}$, and the respective errors in the evaluation of the correlation function are of the order $3 \times 10^{-3}$. Therefore, the decay observed in Figure 1 represents a real effect. The correlation function for the 729 cell cycleregulated genes (Spellman et al., 1998) in Figure 1A shows a clear periodic behavior superimposed on a slow decay. Although the number of experimental points is small, a possible upper limit for the decay of auto-correlation can be estimated as $C(t) \approx \mathrm{t}^{-2 / 5}$. This implies that the system does not reach equilibrium in observable time unless the non-exponential decay is followed by an exponential decay phase. If the pure non-exponential decay is maintained, the precision limit of $10^{-3}$ for the function $C(t)$ is reached for $\mathrm{t} \approx 715,000$ years.
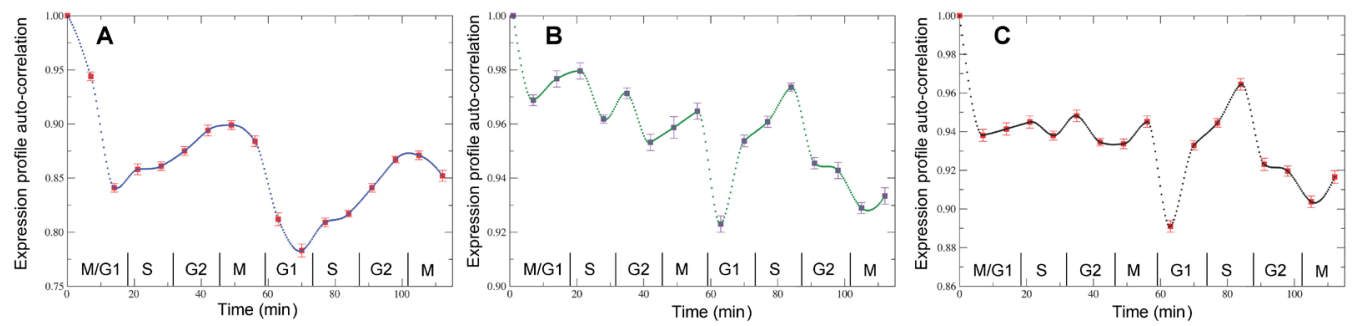

Figure 1. Expression profile auto-correlation functions for the yeast whole-genome. A. Profile auto-correlation of the set of cell cycle-regulated genes ( 729 genes). Red boxes represent the microarray measurements and the blue dotted line is the interpolation of experimental data. B. Genome-wide expression correlation decay excluding the cell cycle-regulated genes (5067 genes). Purple boxes represent microarray measurements and the green dotted line is the interpolation of experimental data. C. Profile auto-correlation of the set of 1651 genes that minimize noise (Ferreira et al., 2007). Red boxes represent microarray measurements and the black dotted line is the interpolation of experimental data.

The auto-correlation function in Figure $1 \mathrm{~A}$ also shows a clear separation of the cell cycle phases G1, S, G2 and M. During G1 and M, the subsystem of cell cycle-regulated genes decorrelates. S and G2 correspond to an increase in coherence whose end points are 
close to a short plateau. Another remarkable fact about this subsystem is the minimum of the auto-correlation function around the 10th array (around $70 \mathrm{~min}$ ), which appears approximately 7 min later than the minimum of the auto-correlation for the rest of the genome (Figure 1B). Figure 1B shows the auto-correlation function of global expression for 5067 non-cell cycle-regulated genes. Although slow, the decay of the correlation function is clear. Inside the first cell cycle, we observe short oscillations in close correspondence to the cell cycle phases. However, this correspondence includes a shift of $\sim 7$ min relative to the decay of cell cycle-regulated genes. Among these $\sim 5000$ genes, we observe a subgroup of 1651 genes that minimize noise (Ferreira et al., 2007). The correlation function for these genes decays very fast in the first minutes of the cell cycle and then stabilizes, indicating that these genes reach a stationary state within the cell cycle (Figure 1C). This shows that the basis of "noise" is strongly dependent on specific groups of genes.

This collective behavior of gene expression patterns reveals robust properties of the cell population and implies that the cell-to-cell variations in mRNA expression are enhanced by the collective decoherence process. Our observation on how the global expression profiles behave with time shows that stochastic fluctuations are not just observed cell-to-cell but also collectively. Moreover, cell-to-cell variation of expression profiles is significantly influenced by decoherence. Therefore, we do not discriminate different sources of expression fluctuations (intrinsic or extrinsic) (Shahrezaei et al., 2008). Gene expression fluctuations are intrinsic properties of collectivities of cells because these fluctuations are not damped in time. The process of decoherence is sufficiently slow to allow the conjecture that transcriptional "noise" is a real emergent property of cell populations and should be treated as such. The slow decay of correlations is a clear signature of strong dependence on the dynamics of transcription of different genes. We conjecture that, in fact, the populational variation in gene expression is a consequence of the continuous decoherence process taking place in evolution.

In conclusion, our central idea is that gene expression noise is a consequence of a decoherence process. It is important to stress that we do not refer to quantum decoherence, a concept very well established in the community of physicists. What we assume is that decoherence is related to the natural loss of synchronization of cell populations due to stochastic fluctuations in cell cycle length. Gene expression noise is currently viewed and treated as a mere populational phenomenon. As far as we know our study is the first to analyze noise in the whole genome using the idea of temporal noise and decoherence to explain the basis of gene expression noise.

\section{ACKNOWLEDGMENTS}

Research supported by grants from the Howard Hughes Medical Institute, FAPESP and CNPq (Brazil).

\section{REFERENCES}

Cho RJ, Campbell MJ, Winzeler EA, Steinmetz L, et al. (1998). A genome-wide transcriptional analysis of the mitotic cell cycle. Mol. Cell 2: 65-73.

Ferreira RC, Bosco F, Paiva PB and Briones MR (2007). Minimization of transcriptional temporal noise and scale invariance in the yeast genome. Genet. Mol. Res. 6: 297-314.

Nacher JC and Akutsu T (2006). Sensitivity of the power-law exponent in gene expression distribution to mRNA decay rate. Physics Letters A 360: 174-178. 
Paulsson J (2005). Models of stochastic gene expression. Physics Life Rev. 2: 157-175.

Raser JM and O'Shea EK (2005). Noise in gene expression: origins, consequences, and control. Science 309: 2010-2013.

Shahrezaei V, Ollivier JF and Swain PS (2008). Colored extrinsic fluctuations and stochastic gene expression. Mol. Syst. Biol. 4: 196.

Spellman PT, Sherlock G, Zhang MQ, Iyer VR, et al. (1998). Comprehensive identification of cell cycle-regulated genes of the yeast Saccharomyces cerevisiae by microarray hybridization. Mol. Biol. Cell 9: 3273-3297. 